\title{
ERRATUM
}

\section{State pension contributions and fiscal stress - ERRATUM}

\author{
DAVID SPLINTER
}

doi: 10.1017/S1474747215000189, Published online by Cambridge University Press 25 August 2015.

Please note the asterisks in Tables 2, 4 and A1 are incorrect. The Tables should have appeared as follows:

Table 2. Pension contribution ratios and expected contribution cuts

\begin{tabular}{|c|c|c|c|c|c|c|}
\hline \multirow[b]{2}{*}{ Unexpected Deficit (\$pc) } & \multicolumn{4}{|c|}{ Pension Contribution Ratios } & \multicolumn{2}{|c|}{$\begin{array}{c}\text { Expected } \\
\text { Contribution Cuts }\end{array}$} \\
\hline & $-0.064^{* * *}$ & $(0.023)$ & $-0.052^{* *}$ & $(0.024)$ & $-0.0073^{*}$ & $(0.004)$ \\
\hline Unexpected Surplus (\$pc) & 0.009 & $(0.016)$ & -0.002 & $(0.015)$ & -0.001 & $(0.001)$ \\
\hline $\begin{array}{l}\text { Lagged End-of-Year Balance } \\
(\$ \mathrm{pc})\end{array}$ & 0.010 & $(0.008)$ & 0.003 & $(0.005)$ & $0.002^{*}$ & $(0.001)$ \\
\hline Public Employees & 0.7 & $(1.2)$ & 0.5 & $(2.8)$ & 0.01 & $(0.18)$ \\
\hline Public Union Members & -2.3 & (2.9) & 9.1 & $(5.7)$ & -0.12 & $(0.40)$ \\
\hline Lagged Funding Ratio & 0.92 & $(0.14)$ & 0.18 & $(0.13)$ & & \\
\hline Tax Limit & -0.1 & $(4.5)$ & 0.3 & (3.6) & -0.3 & $(0.3)$ \\
\hline Constant & $82.4^{* * *}$ & $(15.0)$ & $49.2^{* *}$ & $(20.9)$ & -0.6 & $(1.1)$ \\
\hline State Fixed Effects & NO & & YES & & YES & \\
\hline $\mathrm{R}$-squared & 0.038 & & 0.452 & & 0.153 & \\
\hline Observations & 507 & & 507 & & 952 & \\
\hline
\end{tabular}

Notes: Significance ${ }^{* *} p<0.01, * * p<0.05,{ }^{*} p<0.10$. 
Table 4. Robustness checks: unexpected deficits and state pension contribution ratios

\begin{tabular}{lcccccc}
\hline \hline & \multicolumn{2}{c}{ Fractional Logit } & \multicolumn{2}{c}{ Annual Trend } & \multicolumn{2}{c}{$1992-2009$} \\
\hline Unexpected Deficit (\$pc) & $-0.038^{* * *}$ & $(0.012)$ & $-0.050^{*}$ & $(0.025)$ & $-0.043^{* *}$ & $(0.019)$ \\
Unexpected Surplus (\$pc) & -0.008 & $(0.014)$ & -0.013 & $(0.015)$ & 0.0003 & $(0.014)$ \\
Lagged End-of-Year & 0.001 & $(0.005)$ & $0.016^{*}$ & $(0.008)$ & 0.002 & $(0.005)$ \\
$\quad$ Balance (\$pc) & & & & & & \\
Public Employees & 0.2 & $(2.2)$ & 0.5 & $(2.6)$ & 0.4 & $(2.4)$ \\
Public Union Members & 7.4 & $(4.9)$ & 6.3 & $(5.8)$ & $9.7^{* *}$ & $(4.8)$ \\
Lagged Funding Ratio & 0.12 & $(0.12)$ & 0.21 & $(0.14)$ & $0.24^{*}$ & $(0.14)$ \\
Tax Limit & 1.8 & $(3.1)$ & 5.9 & $(3.7)$ & -0.7 & $(4.3)$ \\
Annual Trend & & & $-1.0^{* *}$ & $(0.4)$ & & \\
Constant & & & $58.4^{* * *}$ & $(20.2)$ & $44.1^{* *}$ & $(20.0)$ \\
State Fixed Effects & YES & & YES & & YES & \\
R-squared & & & 0.470 & & 0.420 & \\
Log pseudolikelihood & -113.6 & 507 & 507 & & 601 & \\
Observations & 507 & \multicolumn{7}{c}{} \\
\hline \hline
\end{tabular}

Notes: Significance ${ }^{* * *} p<0.01,{ }^{* *} p<0.05,{ }^{*} p<0.10$.

Table A1. Year fixed effects: unexpected deficits and state pension contribution ratios

\begin{tabular}{|c|c|c|c|c|}
\hline \multirow[b]{2}{*}{ Unexpected Deficit (\$pc) } & \multicolumn{2}{|c|}{ Year Fixed Effects } & \multicolumn{2}{|c|}{$\begin{array}{l}\text { Year FE \& Budget } \\
\text { Balance Strength }\end{array}$} \\
\hline & $-0.049^{* *}$ & $(0.024)$ & $-0.047^{* *}$ & $(0.023)$ \\
\hline Unexpected Surplus (\$pc) & 0.001 & $(0.018)$ & -0.005 & $(0.018)$ \\
\hline Lagged End-of-Year Balance (\$pc) & 0.012 & $(0.008)$ & $0.015^{*}$ & $(0.008)$ \\
\hline Public Employees & $1.8^{*}$ & $(1.0)$ & $2.1^{* *}$ & $(1.0)$ \\
\hline Public Union Members & $-2.1^{* *}$ & $(1.1)$ & $-3.0^{* * *}$ & $(1.2)$ \\
\hline Lagged Funding Ratio & 0.11 & $(0.08)$ & $0.13^{*}$ & $(0.08)$ \\
\hline Tax Limit & -0.2 & $(3.0)$ & -0.8 & $(3.0)$ \\
\hline Balanced Budget Strength & & & $-1.1^{*}$ & $(0.58)$ \\
\hline Constant & $72.3^{* * *}$ & $(7.7)$ & $79.8^{* * *}$ & $(8.3)$ \\
\hline Year Fixed Effects & YES & & YES & \\
\hline $\mathrm{R}$-squared overall & 0.033 & & 0.031 & \\
\hline Observations & 495 & & 495 & \\
\hline
\end{tabular}

Notes: Significance ${ }^{* * *} p<0.01,{ }^{* *} p<0.05,{ }^{*} p<0.10$.

\section{Reference}

Splinter, D. State pension contributions and fiscal stress. Journal of Pension Economics and Finance. Published online by Cambridge University Press 25 August 2015, doi: 10.1017/ S1474747215000189. 